\title{
A simple monochromatic flow cytometric assay for assessment of intraerythrocytic development of Plasmodium falciparum
}

\author{
Kasem Kulkeaw ${ }^{1 *} \mathbb{D}$, Nutpakal Ketprasit ${ }^{2}$, Anchalee Tungtrongchitr ${ }^{1}$ and Duangdao Palasuwan ${ }^{2^{*}}$
}

\begin{abstract}
Background: Gold standard microscopic examination of Plasmodium falciparum intraerythrocytic stage remains an important process for staging and enumerating parasitized erythrocytes in culture; however, microscopy is laborious and its accuracy is dependent upon the skill of the examiner.

Methods: In this study, ViSafe Green (VSG), which is a nucleic acid-binding fluorescent dye, was used for assessing in vitro development of $P$. falciparum using flow cytometry.

Results: Fluorescence intensity of VSG was found to depend on the developmental stage of parasites. Specifically, multiple-nuclei-containing schizonts were observed in the VSG ${ }^{\text {high }}$ population, and growing trophozoites and ringshaped forms were observed in the VSG $G^{\text {intermediate }}$ and VSG low populations. The efficacy of VSG-based assay was found to be comparable to the microscopic examination method, and it demonstrated an ability to detect as low as $0.001 \%$ of the parasitaemia estimated by Giemsa staining. Moreover, when applying VSG for anti-malarial drug test, it was able to observe the growth inhibitory effect of dihydroartemisinin, the front-line drug for malaria therapy.
\end{abstract}

Conclusions: Taken together, the results of this study suggest the VSG-based flow cytometric assay to be a simple and reliable assay for assessing P. falciparum malaria development in vitro.

Keywords: Malaria parasite development, Monochromatic flow cytometric assay, Plasmodium falciparum, VSG

\section{Background}

Plasmodium falciparum remains a wide-spreading and highly virulent parasitic protozoan worldwide [1]. The mortality rate is highest in tropical and subtropical areas. Despite the development of effective anti-malarial drugs, drug-resistant strains of malaria are reported annually [1, 2], which emphasizes the need for ongoing drug resistance surveillance, continued study of the underlying mechanisms of drug resistance, and novel drug development. Culture of laboratory strains or field-isolates of

\footnotetext{
*Correspondence: kasem.kuk@mahidol.edu; nantadao@gmail.com 1 Department of Parasitology, Faculty of Medicine Siriraj Hospital, Mahidol University, 2, Wanglang Road, Bangkoknoi, Bangkok 10700, Thailand ${ }^{2}$ Oxidation in Red Cell Disorders and Health Research Unit, Department of Clinical Microscopy, Faculty of Allied Health Sciences, Chulalongkorn University, 154 Rama 1 Road, Pathumwan, Bangkok 10330, Thailand
}

P. falciparum has been widely used for these investigations. Microscopic examination is an effective method for assessing in vitro growth of malaria parasites in $P$. falciparum culture, as well as for drug sensitivity testing [3-5]. Nevertheless, the counting of malaria-infected erythrocytes under a microscope is tedious and timeconsuming. This method requires a well-trained and experienced microscopist to enumerate and differentiate various stages of malaria parasites. Inter-rater variability among microscopists is, therefore, a drawback of the microscopic examination method.

Flow cytometry facilitates quantitative analysis of cells at high-speed, at high sensitivity, and in a semiautomatic manner. Given that erythrocytes lack nuclear DNA, detection of malarial DNA in erythrocytes is one of the most common assays. Several 
DNA-binding fluorescent dyes (fluorochromes) are available. Some fluorochromes, including hydroethidine [6], ethidium bromide [7], propidium iodide [8], SYBR Green I [9, 10], YOYO-1 [11], Hoechst 33258 [12], and Hoechst 33342 [13], are employed to stain the DNA of the malaria parasite in erythrocytes. To use hydroethidine and Hoechst 33342, cells need to be incubated at $37{ }^{\circ} \mathrm{C}$, which lengthens the processing time. The use of ethidium bromide has decreased due to its carcinogenic property. Propidium iodide was useful for evaluating invasion of merozoites into erythrocytes in anti-malarial drug test [8]. SYBR Green I facilitated quantification of parasitized erythrocytes at different stages of development [9]. However, propidium iodide, SYBR Green I, YOYO-1, and Hoechst require an extra step of cell membrane permeabilization for which aldehyde-based or ethanol-based fixation is often used. Since these methods alter cell structure, morphologic study of malaria parasite cannot be performed after their use.

Many nucleic acid-binding fluorochromes are commercially available and have been applied for visualizing DNA or RNA in agarose or polyacrylamide gel. ViSafe Green (VSG) is a stable, sensitive, and environmentally safe nucleic acid-binding fluorescent dye. VSG can be activated by a $250-300 \mathrm{~nm}$ wavelength (UV) and it emits spectra similar to that of ethidium bromide [14]. Thus, VSG is an alternative to ethidium bromide for visualizing DNA or RNA in agarose gel. Given the availability of new nucleic acid-binding fluorochromes, this study set forth to develop a simple and fixation-free method that uses VSG to enumerate malaria-infected erythrocytes, and to assess intraerythrocytic development in culture. In addition, its utility for anti-malarial drug susceptibility assay was demonstrated.

\section{Methods}

\section{Parasite and culture}

Plasmodium falciparum strain $\mathrm{K} 1$ was used in this study. Parasites were maintained as described previously [15]. Briefly, malaria culture medium (MCM) was prepared that consisted of RPMI 1640 (Sigma-Aldrich Corporation, St. Louis, MO, USA) supplemented with $5.96 \mathrm{~g} / \mathrm{L}$ HEPES, $2 \mathrm{~g} / \mathrm{L}$ sodium bicarbonate, and 10\% heat-inactivated human $A B$ serum. The parasites were cultured in a T-25 flask containing 5\% human O+ erythrocytes in MCM in a $5 \% \mathrm{CO}_{2}$ environment at $37^{\circ} \mathrm{C}$. To assess the developmental stages of the studied parasites, a thin blood smear was prepared on a glass slide. Cells were visualized by staining with Giemsa dye prior to observation under light microscope [16].

\section{Synchronization of Plasmodium falciparum-infected erythrocytes}

Parasites were maintained in a synchronicity manner as described previously [17]. Briefly, parasites were allowed to grow to the ring stage, and they could not be older than 10 to $12 \mathrm{~h}$ after merozoite invasion. The parasite culture was spun down at 2000 revolutions per minute (rpm) for $5 \mathrm{~min}$. After removal of the supernatant, an equal volume of sterile $5 \% \mathrm{D}$-sorbitol in distilled water was mixed with the packed erythrocytes and the mixture was incubated at $37^{\circ} \mathrm{C}$ for $10 \mathrm{~min}$. After incubation, the cell suspension was spun down at $2000 \mathrm{rpm}$ for $5 \mathrm{~min}$ and then washed three times with RPMI 1640. The parasitaemia and synchronicity were evaluated by counting the infected cells per 1000 erythrocytes on a Giemsa-stained thin blood smear under a microscope. Ninety percent synchronicity was accepted for this experiment. The synchronized parasites were adjusted to $1 \%$ parasitaemia with fresh human $\mathrm{O}+$ erythrocytes and cultured in MCM as described above.

\section{Preparation of $P$. falciparum gametocyte}

Gametocytes were prepared as described previously [18]. Briefly, the parasites were allowed to grow to the ring stage at $3-5 \%$ parasitaemia in MCM and then adjusted to $1 \%$ ring-stage parasitaemia with fresh human $\mathrm{O}+$ erythrocytes. To induce gametocyte formation, MCM was replaced with a gametocyte-inducing medium, which is MCM consisting of $0.37 \mathrm{mM}$ hypoxanthine (Sigma-Aldrich) and 10\% human $\mathrm{AB}$ serum without heat inactivation. A $75 \%$ volume of the gametocyte-inducing medium was replaced daily. To assess sexual development of $P$. falciparum, a thin blood smear was prepared on a glass slide and stained with Giemsa dye prior to observation under a light microscope. Gametocytes were identified as described in a published method [19].

\section{ViSafe Green staining and flow cytometric analysis}

Given that no previous study has used VSG $(20 \mathrm{mg} / \mathrm{mL}$; Vivantis Technologies, Salangor, Malaysia) for nucleic staining in viable cells, the fluorescent dye concentration was initially optimized. Briefly, cells were suspended in diluted concentrations of VSG $(0.5,1,2,5$, 10 , and $20 \mu \mathrm{g} / \mathrm{mL}$ ) in phosphate-buffered saline (PBS) and kept in the dark at room temperature (RT) for $20 \mathrm{~min}$. Cells were then subjected to flow cytometric analysis and cell sorting using a FACS Aria II Instrument (BD Biosciences, San Jose, CA, USA) without cell washing. A threshold of FSC was set at 10,000 to reduce a contamination of cell debris (Additional file 1: Fig. S1). A type of VSG-activating laser and a suitable fluorescence detector of an emitted fluorescent 
signal were determined. Given that the FACS Aria II is equipped with 488-, 633-, and 375-nm lasers, all three lasers were used for VSG activation. Fluorescence detectors of FITC (500-560 nm), PE (543-627 nm), PETexas Red (593-639 nm), PerCP-Cy5-5 (655-735 nm), PE-Cy7 (720-840 nm), APC (640-680 nm), A700 (685$775 \mathrm{~nm})$, APC-Cy7 (720-840), BV421 (400-500 nm), BV510 (500-560 nm), and BV605 (590-630 nm) were used for detection of the emitted fluorescent signals. The flow cytometric data were analysed using FlowJo version 10 software (Tree Star, Inc., Ashland, OR, USA). To increase the accuracy of flow cytometric analysis, non-single cells were excluded by gating according to forward scatter (FSC) and side scatter (SSC) characteristics of cells. Briefly, cells were first gated using forward scatter area (FSC-A) parameter at the $\mathrm{X}$-axis, and then using forward scatter height (FSC-H) parameter at the Y-axis. Cells having the characteristic of FSC-A equal to FSC-H were gated. Then, side scatter width (SSC-W) and side scatter height (SSC-H) were set at the $\mathrm{X}$-axis and $\mathrm{Y}$-axis, respectively, in order to exclude cells having SSC-W $\mathrm{W}^{\text {high }}$, which are not single cells. Cells were then further gated according to forward scatter width (FSC-W) and forward scatter height (FSC-H). Cells were sorted into PBS containing 1\% fetal bovine serum (FBS) for morphologic analysis.

\section{Giemsa staining and microscopy}

Cells were affixed to glass slides using a CytoSpinTM4 Cytocentrifuge (Thermo Fisher Scientific, Inc., Waltham, MA, USA) at $450 \mathrm{rpm}$ for $7 \mathrm{~min}$, and then rapidly airdried. Cells were fixed with absolute methanol and stained using a 1:18 diluted Giemsa solution at RT for $30 \mathrm{~min}$. After one wash with running tap water, slides were air-dried and covered with glass coverslips with one drop of mounting solution. Cell morphology was assessed using an Olympus BX53 using an objective lens at 100× magnification. For Giemsa-stained thin film of the culture, a minimum of 100 fields was examined at $100 \times$ magnification with oil immersion [20].

\section{Fluorescence microscopic imaging}

To ensure that VSG is able to pass through the cell membrane and bind to the parasite's nucleic acid, 50-100 $\mu \mathrm{L}$ of VSG-stained cells was dropped onto a glass slide and covered with a thin glass. The VSG-stained cells were observed under a laser-scanning confocal microscope (ECLIPSE Ti-Clsi4 Laser Unit; Nikon Corporation, Tokyo, Japan). Differential interference contrast and a 488-nm argon-ion laser was used for microscopic imaging.

\section{Reliability and sensitivity}

To test the reliability of the VSG-based flow cytometric assay, the parasitaemia estimated from microscopic examination of the Giemsa-stained blood smear (the standard method) and the percentage of VSG+ cells obtained from flow cytometry were compared. Various concentrations of parasitaemia were prepared by diluting the parasitized erythrocytes in a 5\% uninfected erythrocyte suspension. Spearman's rank correlation coefficient was used to assess the strength of association between the standard microscopic assay and VSG-based flow cytometry. For sensitivity testing, culture of P. falciparum was diluted to $0.001 \%$ parasitaemia, which is the limit of detection in routine microscopic diagnosis [20], and then stained with VSG as describe above and analysed by flow cytometry.

Lethal induction of $P$. falciparum using dihydroartemisinin Dihydroartemisinin (DHA) (Sigma-Aldrich), which is a primary drug for falciparum malaria treatment, was used in this study to induce lethal form of the parasites.

DHA was prepared at a concentration of $700 \mathrm{nM}$ in dimethyl sulfoxide (DMSO) (Sigma-Aldrich) as described in previous study [21]. In short, $2 \mathrm{mg}$ of DHA was resuspended in $2 \mathrm{~mL}$ of DMSO and used as stock solution. The stock solution was then diluted fivefold in DMSO to achieve a drug concentration of $200 \mu \mathrm{g} / \mathrm{mL}(700 \mu \mathrm{M})$. Synchronized ring stages of $P$. falciparum $\mathrm{K} 1$ strain were diluted with $5 \%$ haematocrit $\mathrm{O}$ cell and MCM to obtain $1 \%$ parasitaemia and $2 \%$ haematocrit. Twenty $\mu \mathrm{L}$ of DHA solution $(700 \mu \mathrm{M})$ or DMSO was mixed with $2 \mathrm{~mL}$ of $\mathrm{MCM}$ to obtain a concentration of $7 \mu \mathrm{M}$. Then, $100 \mu \mathrm{L}$ of $7-\mu \mathrm{M}$ DHA was mixed with $900 \mu \mathrm{L}$ of the infected erythrocytes. Therefore, the final concentration of DHA in the culture was $700 \mathrm{nM}$. To examine dose-dependent effect of DHA, three different concentrations of DHA (350, 700 and $1400 \mathrm{nM}$ ) were prepared. The parasites were then exposed to DHA or DMSO (as control) in a $5 \% \mathrm{CO}_{2}$ atmosphere at $37^{\circ} \mathrm{C}$ for $24 \mathrm{~h}$. To access time-dependent effect of DHA, the parasites were exposed to 700-nM DHA for $6 \mathrm{~h}$ and then cultured in MCM without DHA [21]. The parasites were collected at different time points (12, 24, 36, 48 and $60 \mathrm{~h}$ ) and subjected to Giemsa dye stain and VSG-based flow cytometry as described above.

\footnotetext{
Statistical analysis

Data analysis and graph generation were performed using GraphPad Prism software version 5.0 (GraphPad Software, Inc., San Diego, CA, USA). Results are expressed as mean \pm standard deviation (SD) and coefficient of variation $(\mathrm{CV})$. Spearman's rank correlation coefficient was used to measure the strength of association between
} 
standard microscopy and VSG-based flow cytometry. Statistically significant differences were identified using non-parametric Student's $t$-test. A $p$-value less than 0.05 was regarded as being statistically significant.

\section{Results}

\section{Cell permeability of VSG dye}

To ensure that VSG is cell-permeable and that it binds to nucleic acid, a non-synchronized culture of $P$. falciparum (Fig. 1a) was incubated with VSG dye without fixation and subjected to laser-scanning confocal microscope imaging in which the emitted fluorescent signal of VSG was displayed as green. To deny the possibility of autofluorescence, a sample of unstained $P$. falciparuminfected erythrocytes was used as a control. There was no green colour observed in the control (Fig. 1b, lower panels). At lower magnification, cells having green colour were observed, and they accounted for $1.9 \%$ of total observed cells (Fig. 1b, upper panels). Higher magnification images revealed green colour inside the erythrocytes (Fig. 1b, yellow and blue arrows in middle panels), which suggests cell membrane permeability of VSG. Moreover, the intensity of green colour was shown to vary, with intensity roughly grouped into low or high intensity (Fig. 1b, yellow and blue arrows, respectively). Two green dots were also observed in a single erythrocyte similar
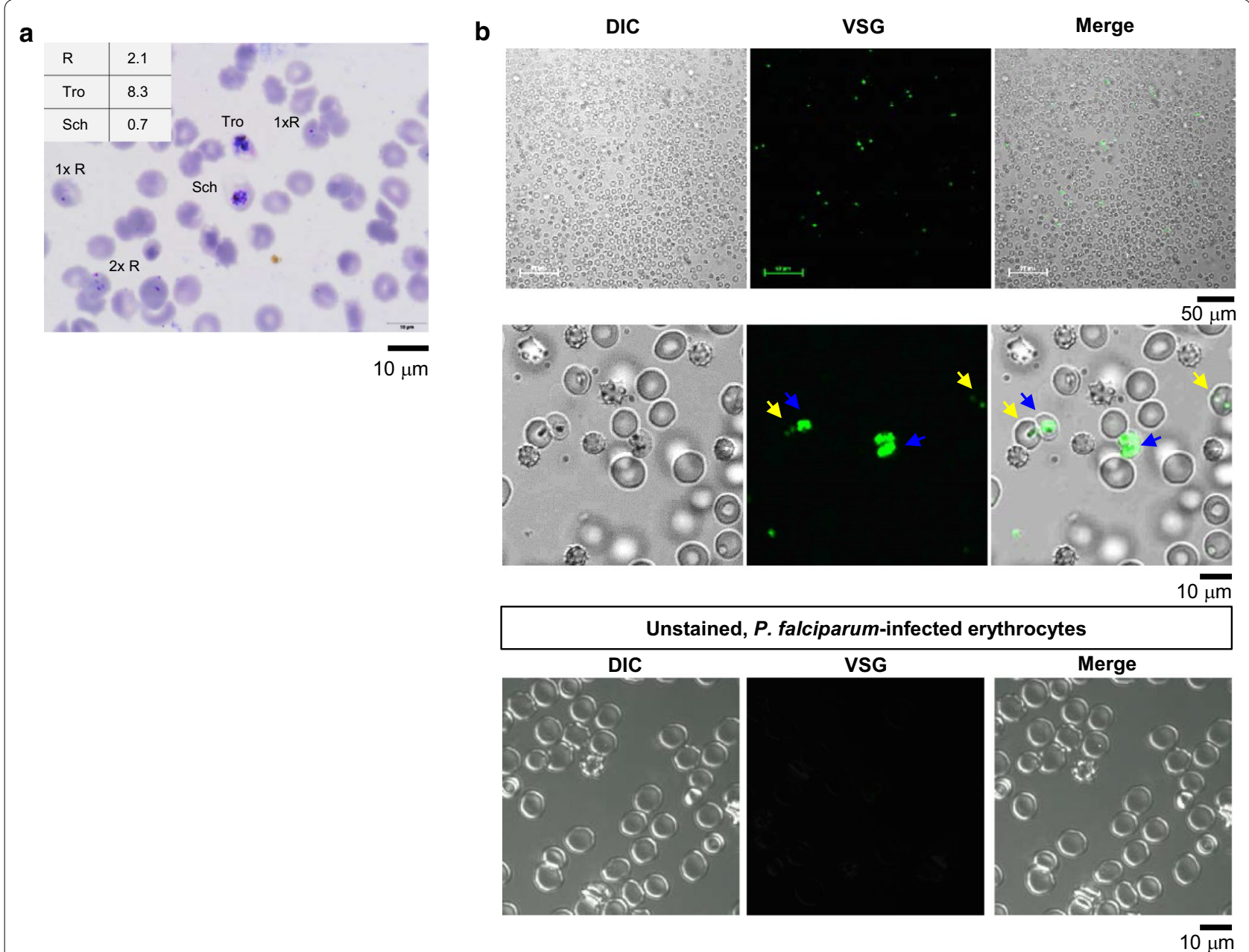

Fig. 1 Cell permeability of VSG dye. a Giemsa-stained thin blood smear of the non-synchronized culture revealed mixed developmental stages of falciparum malaria parasites. Scale bars: $10 \mu \mathrm{m}$. b Laser scanning confocal microscopic images of P. falciparum-infected erythrocytes uptaking VSG (upper and middle panels). Differential interference contrast images are shown (far left panel). VSG was activated by a 488-nm argon-ion laser, and is displayed as green in the middle panel. Differential interference contrast and fluorescent images are merged in the far-right panel. Yellow and blue arrows indicate low and high intensity of VSG, respectively. The unstained, P. falciparum-infected erythrocytes were used as a control. Scale bars: $50 \mu \mathrm{m}$ at upper and $10 \mu \mathrm{m}$ at middle and lower panels. R, ring form; Tro, trophozoite; Sch, schizont; DIC, differential interference contrast; VSG, ViSafe Green 
to those found in the Giemsa-stained thin blood smear, which suggests multiple infection of $P$. falciparum. These findings indicate that VSG was able to permeate the $P$. falciparum-infected erythrocytes.

\section{Optimization of VSG stain for flow cytometry}

Given that VSG has never been used for flow cytometry, a type of VSG-activating laser and a suitable fluorescence detector had first to be identified. The concentration of VSG was then optimized. In flow cytometry analysis, non-single cells were excluded by gating according to forward scatter (FSC) and side scatter (SSC) characteristics of cells. Briefly, cells were first gated using FSC-A parameter at the $\mathrm{X}$-axis, and using $\mathrm{FSC}-\mathrm{H}$ parameter at the Y-axis (Fig. 2a, upper panel). Cells having the characteristic of FSC-A equal to FSC-H were gated. Then,

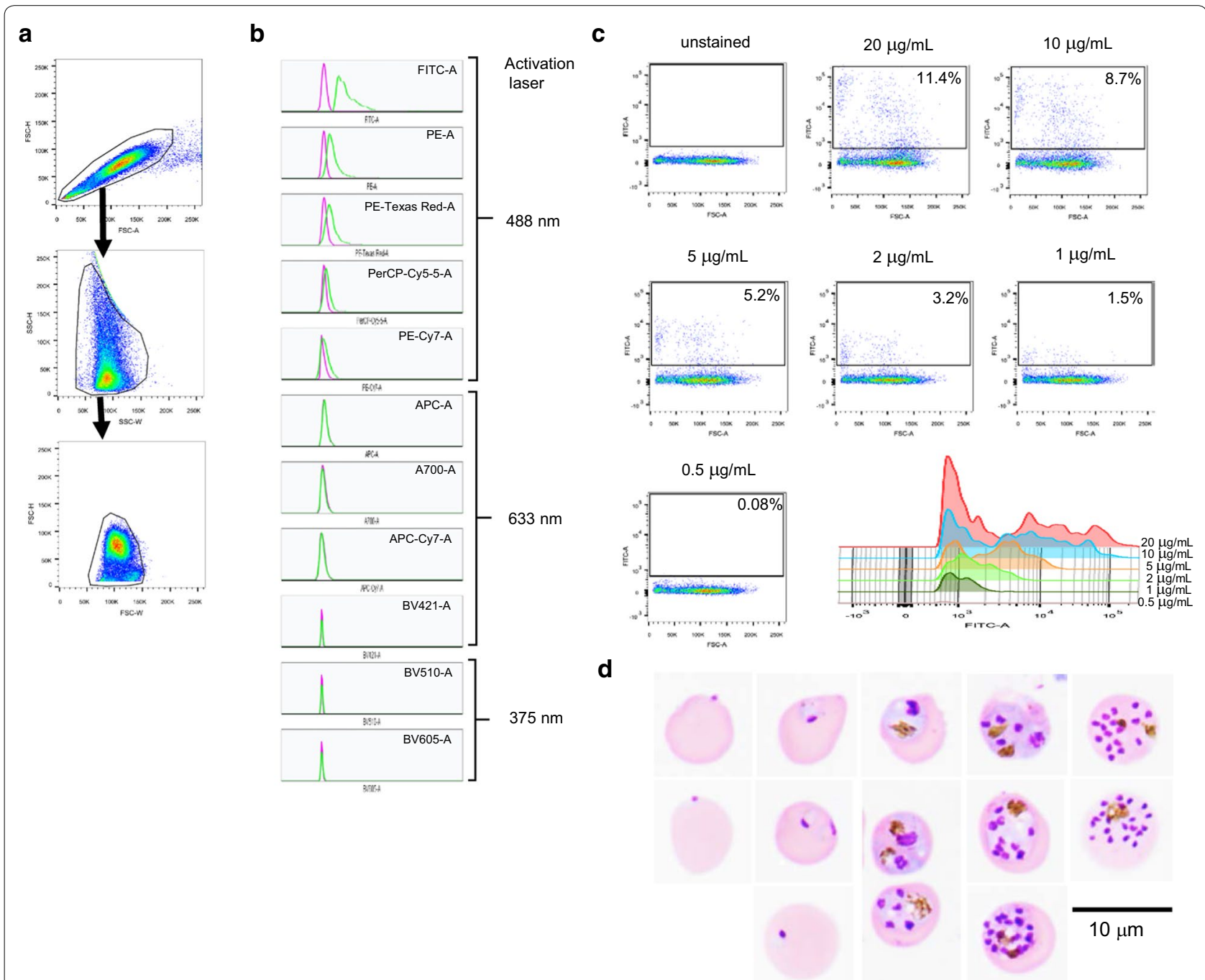

Fig. 2 Optimization of VSG staining of P. falciparum-infected erythrocytes. a Gate setting for flow cytometric analysis. Non-single cells were excluded by gating according to FSC-H, FSC-W, SSC-H, and SSC-W. b Histograms show fluorescence intensity of VSG + cells (green) excited by 488-nm, 633-nm, and 375-nm lasers. To read the emitted fluorescent signal, detectors of FITC (500-560 nm), PE (543-627 nm), PE-Texas Red (593-639 nm), PerCP-Cy5-5 (655-735 nm), and PE-Cy7 (720-840 nm) were used for 488-nm activating laser; detectors of APC (640-680 nm), A700 (685-775 nm), and APC-Cy7 (720-840) were used for 561-nm activating laser; and, detectors of BV421 (400-500 nm), BV510 (500-560 nm), and BV605 (590-630 nm) were used for 445-nm activating laser. Histogram of sample not stained with VSG was set as VSG negative (shown in magenta). c Representative flow cytometric profiles of samples stained with VSG at $0.5,1,2,5,10$, and $20 \mu \mathrm{g} / \mathrm{mL}$ relative to the 10,000x concentration (20 mg/ $\mathrm{mL}$ ) of the commercial version. Overlaid histogram of VSG+ cells obtained from staining with different concentrations of VSG is shown on the left side of flow cytometric images. $\mathbf{d}$ Representative images of Giemsa-stained erythrocytes in VSG+ fraction acquired using an objective lens at 100x. Cells were sorted from the sample stained with $10 \mu \mathrm{g} / \mathrm{mL}$ of VSG. Scale bars: $10 \mu \mathrm{m}$. FSC-A, forward scatter area; FSC-H, forward scatter height; FSC-W, forward scatter width; SSC-W, side scatter width; SSC-H, side scatter height; DIC, differential interference contrast; VSG, ViSafe Green 
SSC-W and SSC-H were set at the $\mathrm{X}$-axis and Y-axis, respectively (Fig. 2a, middle panel), in order to exclude cells having SSC-W ${ }^{\text {high }}$, which are not single cells. Cells were then further gated according to FSC-W and FSC-H (Fig. 2a, lower panel). Based on FSC-A and SSC-A, there were two populations: cells having FSC-A lower or higher than $50 \mathrm{~K}$ (Additional file 1: Fig. S1A). Both contained $P$. falciparum-infected and non-infected erythrocytes (Additional file 1: Fig. S1B). Thus, both populations were included for analysis. These initial gating steps aimed to obtain single cells, which increases the accuracy of flow cytometric analysis. Using a FACS Aria II, the 488nm laser could activate VSG and effectuate emission of a fluorescent signal, whereas the $633-\mathrm{nm}$ and $375-\mathrm{nm}$ laser could not (Fig. 2b). When a detector of FITC fluorochrome (500-560 nm) was used, VSG+ cells (green-coloured lines) could be separated from the unstained cells (magenta-coloured lines). In contrast, when a detector of PE (543-627 nm) and PE-Texas Red (593-639 nm) was used, VSG+ cells (green-coloured lines) overlapped with the unstained cells (magenta-coloured lines), which limited our ability to analyse the parasitized cells. Therefore, it was decided to use the 488-nm laser for VSG activation, and the FITC detector to read the emitted fluorescent signal.

To determine the optimal concentration of VSG, $P$. falciparum-infected erythrocytes were incubated with $0.5,1,2,5,10$, and $20 \mu \mathrm{g} / \mathrm{mL}$ of VSG. The optimal VSG concentration was determined based on its ability to fractionate $P$. falciparum-infected erythrocytes from non-infected cells. As shown in Fig. 2c, 20 and $10 \mu \mathrm{g} / \mathrm{mL}$ of VSG were the concentrations that yielded the highest fluorescence intensity in VSG+ cells. Moreover, different intensity of fluorescence was observed in the 20 and $10 \mu \mathrm{g} / \mathrm{mL}$ VSG-stained samples (Fig. 2c, histogram), which is a finding that is consistent with confocal microscopic data. The 200,100 , and $50 \mu \mathrm{g} / \mathrm{mL}$ VSG concentrations were excluded due to an upward shift in the dots on the flow cytometric profile, which suggested an increase in non-specific staining (high background). Microscopic observation of sorted VSG+ cells showed that $10 \mu \mathrm{g} / \mathrm{mL}$ of VSG yielded all stages of intraerythrocytic development of P. falciparum (Fig. 2d). In agreement with Fig. 2d, Giemsa staining of presorted sample showed $10.4 \%$ parasitaemia that consisted of $9.8 \%$ ring form, $0.1 \%$ trophozoites, and $0.5 \%$ schizonts, which strongly suggests the accuracy of VSG at a concentration of $10 \mu \mathrm{g} / \mathrm{mL}$. Therefore, $10 \mu \mathrm{g} / \mathrm{mL}$ of VSG was used for other experiments in this study.

\section{Validation of the VSG staining method}

To test that each stage of intraerythrocytic development of P. falciparum could be fractionated based on the intensity of VSG, a non-synchronized culture of malaria parasites was prepared. As a standard method, Giemsa staining of thin blood film showed 14\% parasitaemia that consisted of $13 \%$ ring form, $0 \%$ trophozoites, and $1.1 \%$ schizonts (Fig. 3a). The VSG+ cells were separated according to intensity into low, intermediate, or high (hereafter referred to as VSG ${ }^{\text {low }}$, VSG ${ }^{\text {intermediate, }}$, and VSG ${ }^{\text {high }}$, respectively) (Fig. 3b), and their morphologies were examined. Schizonts were observed only in VSG ${ }^{\text {high }}$ fraction, and ring forms and growing trophozoites were observed only in VSG intermediate $^{-}$ and VSG $^{\text {low }}$ fraction (Fig. 3c). Moreover, different morphology of the $P$. falciparum parasites could be observed in VSG ${ }^{\text {intermediate }}$ and VSG ${ }^{\text {low }}$ fraction. The cytoplasm of $P$. falciparum in the VSG ${ }^{\text {intermediate }}$ fraction was thicker than that in the VSG ${ }^{\text {low }}$ fraction, and it contained malarial pigment (Fig. 3d). These findings were in agreement with microscopically examined Giemsa-stained thin blood film that revealed ring form, trophozoites, and schizonts in the culture, which suggested that this protocol was optimal. Thus, fluorescence intensity of VSG depends on the stage of in vitro malaria development.

To test whether VSG-based flow cytometric analysis could distinguish gametocytes from schizonts, $P$. falciparum strain $\mathrm{K} 1$ was grown in gametocyte-inducing culture medium and performed VSG-based flow cytometric analysis. Cells in VSG ${ }^{\text {low }}$, VSG ${ }^{\text {intermediate }}$, and VSG ${ }^{\text {high }}$ fraction were sorted and stained with Giemsa. In the VSG ${ }^{\text {high }}$ fraction, parasitized erythrocytes could be observed having granular distribution of haemozoin that resembled stage-IB gametocytes. Moreover, some were elongated and D-shaped within erythrocytes, which are key characteristics of stage-II gametocytes. Early schizonts having 2 and 6 divided nucleus, and mature schizonts consisting of 14 merozoites were also observed in the VSG ${ }^{\text {high }}$ fraction, whereas ring forms and trophozoites were observed in the VSG ${ }^{\text {low }}$ and $V_{S G}{ }^{\text {intermediate }}$ fractions, respectively (Additional file 2: Fig. S2). Thus, VSG-based flow cytometric assay is not able to distinguish gametocytes from schizonts.

Given the ability of VSG to differentiate intraerythrocytic stages, the study explored whether change in cell granularity is related to the developmental stages of $P$. falciparum. $\mathrm{VSG}^{\text {low }}, \mathrm{VSG}^{\text {intermediate}}$, and $\mathrm{VSG}^{\text {high }}$ cells were gated and analysed for SSC-A, which is an indicator of cell granularity. As shown in Fig. 3e, the median of SSC-A increased about 2 times when VSG ${ }^{\text {low }}$ and VSG $^{\text {intermediate }}$ cells developed into VSG ${ }^{\text {high }}$ cells. These results suggest that change in cell granularity is related to intraerythrocytic development of P. falciparum, and that this change can be assessed using VSG-based flow cytometry. 


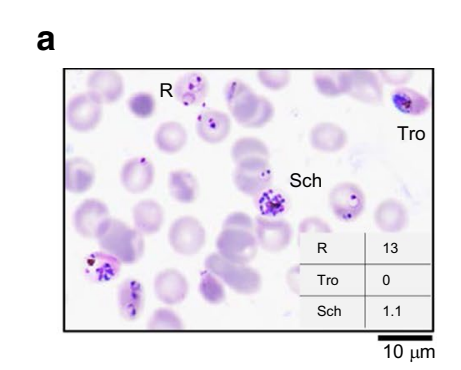

b

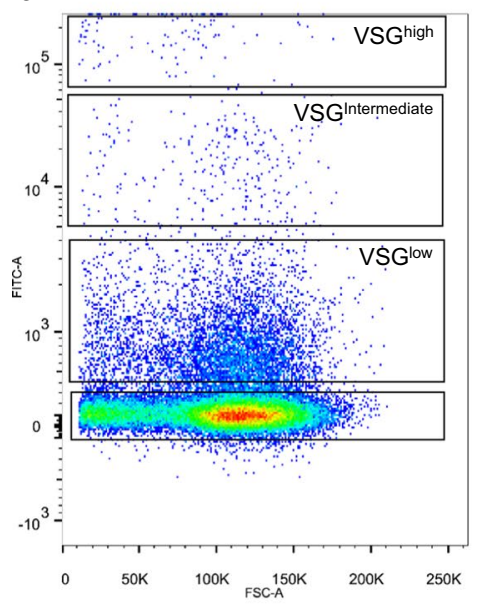

C
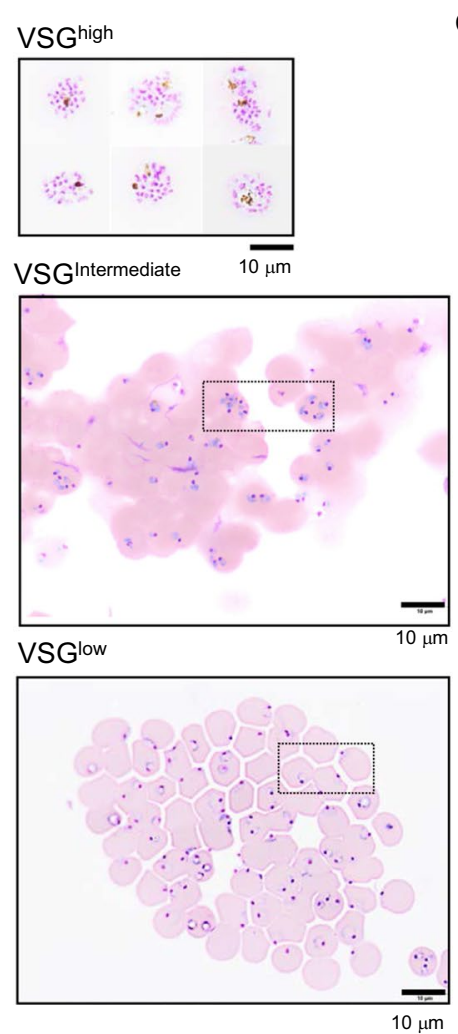

d VSG Intermediate

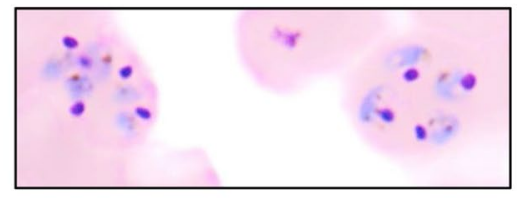

VSGlow

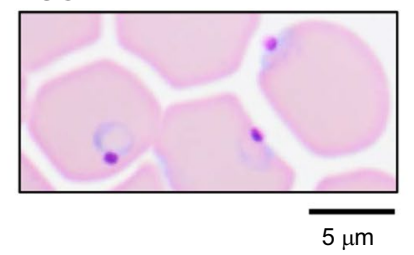

e
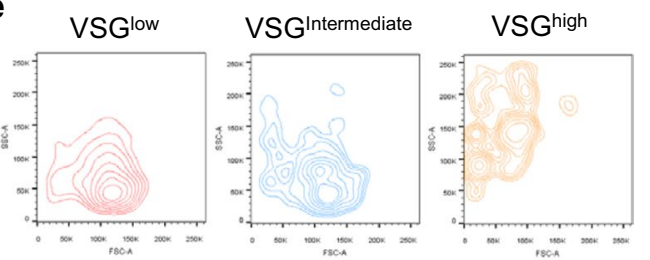

\begin{tabular}{|l|l|c|c|}
\hline \multirow{2}{*}{$\begin{array}{c}V \\
S\end{array}$} & \multirow{2}{*}{$\begin{array}{c}\text { Mean } \\
\text { intensity } \\
\text { of FITC }\end{array}$} & \multicolumn{2}{|c|}{$\begin{array}{c}\text { SSC-A } \\
\text { (Granularity) }\end{array}$} \\
\cline { 3 - 4 } & & Mean & Median \\
\hline & 1,005 & 81,272 & 65,600 \\
\hline & 17,564 & 87,517 & 73,024 \\
\hline & 157,104 & 137,037 & 135,296 \\
\hline
\end{tabular}

Fig. 3 Intensity of VSG depends on the stage of intraerythrocytic development of P. falciparum. a Giemsa-stained thin blood smear of the non-synchronized culture revealed mixed developmental stages of falciparum malaria parasites. $\mathbf{b}$ Flow cytometric profile of $P$. falciparum infected erythrocytes. The non-synchronized culture of malaria parasites was stained with $10 \mu \mathrm{g} / \mathrm{mL}$ of VSG. Single cells were separated based on fluorescence intensity into high, intermediate, or low (hereafter referred to as VSG ${ }^{\text {high }}$, VSG ${ }^{\text {intermediate, }}$, and VSG ${ }^{\text {low, }}$, respectively). c Morphology of Giemsa-stained VSG high, VSGintermediate, and VSGlow cells. $\mathbf{d}$ Higher magnification of P. falciparum-infected cells in the boxed area of VSG ${ }^{\text {intermediate }}$ and VSG low cells in Fig. 3C. e Cell granularity of VSG high, VSG intermediate, and VSGlow cells was assessed based on SSC-A. Scale bars: $10 \mu \mathrm{m}$ for Fig. 3a, C, and $5 \mu \mathrm{m}$ for Fig. 3d. FSC-A, forward scatter area; VSG, ViSafe Green

\section{Linearity and sensitivity of the VSG-based flow cytometric assay}

To evaluate the optimized protocol relative to its ability to enumerate parasitized erythrocytes, detection of malaria-infected erythrocytes was performed in a dosedependent manner. Various concentrations of malariainfected erythrocytes were prepared. Two-fold dilutions of infected cells were prepared using non-infected erythrocytes as diluent. That analysis revealed that VSG-based flow cytometry could detect malaria-infected erythrocytes in a dose-dependent manner (Fig. 4a). The relative values correlated well between the two assays $\left(r^{2}=0.75-\right.$ $0.97 ; p<0.05)$. The same results were observed from three independent experiments $(\mathrm{CV}=11.2 \%)$, indicating the reproducibility of linearity measurement.

To assess the sensitivity of VSG-based flow cytometry, parasitized erythrocytes were diluted to $0.001 \%$, which is the limit of detection in routine microscopic diagnosis [20]. As shown in Fig. 4b, two independent cultures were analysed for each cytometry run. There were $11 \%$ and $9 \%$ parasitaemia enumerated using Giemsa-based microscopy. The parasites were diluted to $0.001 \%$ using non-infected erythrocytes as diluent. The diluted samples having $0.001 \%$ parasitaemia were then subjected to flow cytometry analysis. VSG-based flow cytometry was capable of detecting $0.3 \%$ and $1.1 \%$ of VSG+ cells, which is 300-1000 times higher than the detection rate $(0.001 \%$ parasitaemia) by Giemsa-based microscopy. Next, the reproducibility of the developed assay for enumeration of low parasitaemia was examined. Three independent settings of malaria culture was prepared and diluted them to $0.01 \%$ parasitaemia, which is a minimum value that correlated well with standard microscopic examination (Fig. 4a). All three independent runs of VSG-based flow cytometry were able to detect $0.9 \pm 0.2 \%$ of VSG+ cells $(\mathrm{CV}=22 \%$, Fig. 4c), implying reproducibility comparable to Giemsa-based microscopy $(\mathrm{CV}=21.8 \%)$ for detection of low parasitaemia. 


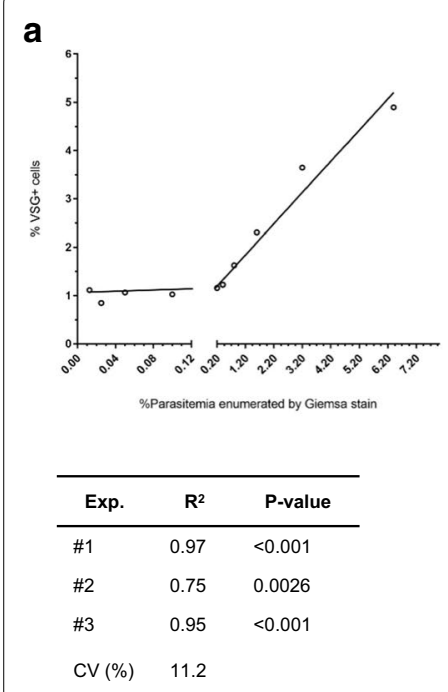

b
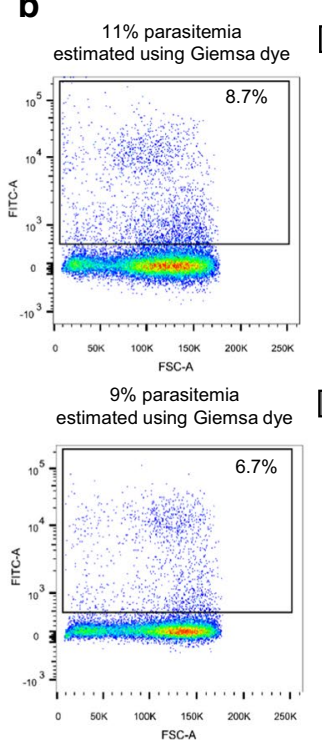

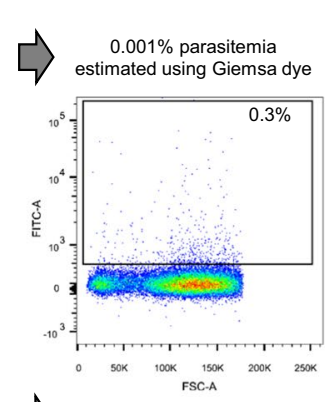

$0.001 \%$ parasitemi
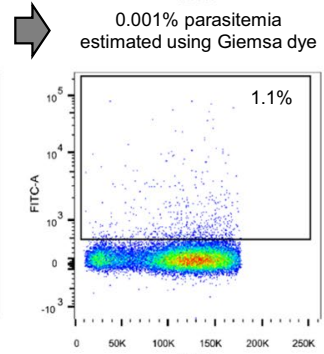

C
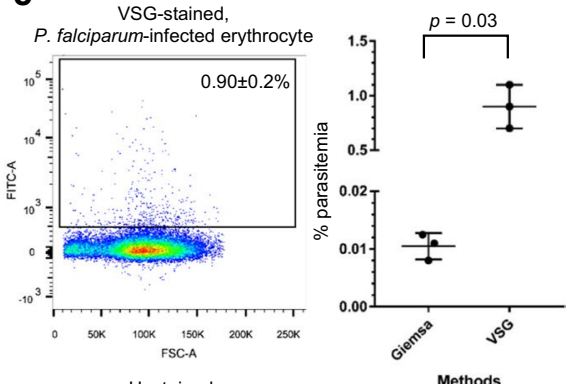

Methods

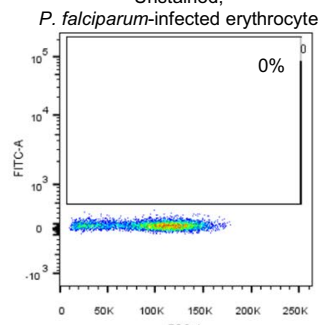

Fig. 4 Linearity and sensitivity of the VSG-based flow cytometric assay. a Representative graph of the Spearman's rank correlation coefficients. Percentage of P. falciparum-infected erythrocytes was obtained from VSG-based flow cytometry (Y-axis) and from Giemsa staining of thin blood film (X-axis). Three independent analyses were performed that revealed a range of infected erythrocytes of $0.01-6.4 \%$, as shown in the table. $\mathbf{b}$ Sensitivity of VSG-based flow cytometry. Culture of P. falciparum was diluted to $0.001 \%$ parasitaemia, which is the limit of detection in routine microscopic diagnosis [20], and then analysed by flow cytometry. Representative flow cytometric profile and data are shown as mean \pm SD. The graph shows a comparison of parasitaemia detected by standard microscope and VSG+ cells detected by flow cytometry. c Reproducibility of the VSG-based flow cytometric assay for low parasitaemia culture. Three independent settings of $P$. falciparum culture were diluted to $0.01 \%$ parasitaemia and analysed using flow cytometry. Unstained, P. falciparum-infected erythrocytes were used as control. VSG, ViSafe Green; FSC-A, forward scatter-area

To examine the variability of VSG-based flow cytometric assay for enumeration and identification of $P$. falciparum-infected erythrocytes among different sets of parasite culture, parasite culture was prepared on different dates and compared the enumerated values of parasitized cells (mean $\pm \mathrm{SD}$ ) obtained from Giemsabased microscopic analysis with those obtained from VSG-based flow cytometric analysis (Table 1). There were

Table 1 Comparison of a standard optical microscope and VSG-based flow cytometer for enumeration and identification of $P$. falciparum-infected erythrocytes

\begin{tabular}{|c|c|c|c|c|c|c|}
\hline \multirow{3}{*}{$\begin{array}{l}\text { Ring-form predominant } \\
\text { culture }\end{array}$} & \multicolumn{6}{|c|}{ Enumerated value of parasitized erythrocyte } \\
\hline & \multicolumn{3}{|c|}{ Microscope } & \multicolumn{3}{|c|}{ VSG-based flow cytometer } \\
\hline & Mean & SD & CV (\%) & Mean & SD & CV (\%) \\
\hline Ring-forms & 11.5 & 2.3 & 20.4 & 10.4 & 3.7 & 35.4 \\
\hline Trophozoites & 0.1 & 0.1 & 141.4 & 1.7 & 1.6 & 91.5 \\
\hline Schizonts & 0.8 & 0.4 & 53.0 & 0.5 & 0.3 & 52.1 \\
\hline All stages & 12.3 & 2.7 & 21.8 & 12.5 & 1.8 & 14.1 \\
\hline \multirow{3}{*}{$\begin{array}{l}\text { Trophozoite predominant } \\
\text { culture }\end{array}$} & \multicolumn{6}{|c|}{ Enumerated value of parasitized erythrocyte } \\
\hline & \multicolumn{3}{|c|}{ Microscope } & \multicolumn{3}{|c|}{ VSG-based flow cytometer } \\
\hline & Mean & SD & CV (\%) & Mean & SD & CV (\%) \\
\hline Ring-forms & 1.1 & 1.5 & 141.4 & 2.7 & 0.2 & 8.0 \\
\hline Trophozoites & 8.5 & 0.3 & 3.3 & 4.4 & 0.4 & 9.6 \\
\hline Schizonts & 0.4 & 0.5 & 141.4 & 0.1 & 0.04 & 60.6 \\
\hline All stages & 9.9 & 1.7 & 17.1 & 7.1 & 0.7 & 9.5 \\
\hline
\end{tabular}


two types of culture: ring-form and trophozoite predominant. In both types of culture, the CV of the VSG-based flow cytometric assay for enumeration of parasitaemia was relatively lower than that of the microscopic method, which implies lower variability of the VSG-based flow cytometric assay. When analysing the variability of assays according to developmental stage, high $\mathrm{CV}$ values were obtained from both Giemsa-based microscopy and VSG-based flow cytometry, which is likely due to low parasitaemia in each developmental stage. Collectively, VSG-based flow cytometry is a reliable, sensitive, and reproducible assay for enumeration of parasitaemia.

\section{Application of VSG-based flow cytometry for synchronicity assessment and drug sensitivity testing}

Synchronization of $P$. falciparum development is a common method used in routine culture, and its aim is to obtain a predominant intraerythrocytic stage of parasites. To explore whether VSG-based flow cytometry is capable of assessing synchronicity of $P$. falciparum development in a routine culture, synchronized and non-synchronized cultures of $P$. falciparum were prepared (Fig. 5a), stained with VSG, and subjected to flow cytometry analysis. Given the ability of flow cytometry to detect cell size and granularity using respective FSC and SSC, it was hypothesized that synchronized parasites have the same size and granularity, which suggests homogeneity. Thus, a quantile contour plot, which is an effective way to visualize distinct populations regardless of the numbers of cells displayed [22], was selected to assess cell homogeneity. In Fig. 5b, only VSG+ cells were displayed based on their size (as indicated by FSC-A on the $\mathrm{X}$-axis) and granularity (as indicated by SSC-A on the Y-axis). To enhance the visualization of a distinct cell population having various cell size and granularity, histograms of FSC-A and SSC-A are also shown at the top and left side of the contour plots, respectively. Given the ability of contour plot to visualize cells based on the relative frequencies of sub-populations, distinct populations of VSG+ cells were able to be located using vertical and horizontal lines drawn on the contour plots. There were at least three distinct populations observed in the nonsynchronized culture (Fig. 5b, left panel), as follows: (1) cells having small size with various granularity (approximately $0-45 \mathrm{~K}$ of FSC-A, and 30-170 K of SSC-A); (2) cells having a relatively large size with high granularity (approximately $45-185 \mathrm{~K}$ of FSC-A, and $75-170 \mathrm{~K}$ of SSC-A); and, (3) cells having a relatively larger size with low granularity (approximately $45-185 \mathrm{~K}$ of FSC-A, and $20-75 \mathrm{~K}$ of SSC-A). In contrast, only one minor (indicated as 1) and one major (indicated as 2) population were observed in the synchronized culture. They had a similar size (50-150 K of FSC-A), but different levels of granularity (35-240 K of SSC-A) (Fig. 5b, right panel). In the left panel of Fig. 5b, the population of VSG+ cells having less than $45 \mathrm{~K}$ of FSC-A was observed only in the non-synchronized culture (indicated as 1), but not in the synchronized culture (Fig. 5b, lower panel). Based on the intensity of VSG and microscopic images (Additional file 3: Fig. S3A), the population number 1, 2, and 3 in the left panel of Fig. 5b are schizonts, trophozoites, and ring forms, respectively. In contrast to observation in the nonsynchronized culture, the minor and major populations of the synchronized culture could be separated based on SSC-A, as follows: (1) minor population with SSC-A higher than $160 \mathrm{~K}$, and (2) major population with SSC-A lower than $160 \mathrm{~K}$ (Fig. 5b, right panel). Compared to the non-synchronized culture, a population of VSG+ cells having SSC-A higher than $160 \mathrm{~K}$ was observed only in the synchronized culture (indicated as 1 in Fig. 5b, lower panel). Based on microscopic images (Additional file 3: Fig. S3B), the VSG+ cells with more than $160 \mathrm{~K} \mathrm{SSC-A}$ are infected erythrocytes containing multiple (60\%) and single $(40 \%)$ ring forms, and they had VSG intensity of 11,578; whereas, the VSG+ cells with lower than $160 \mathrm{~K}$ SSC-A are infected erythrocytes with multiple (35\%) and single (65\%) ring forms. Disappearance of the population having more than $160 \mathrm{~K}$ of SSC-A on the contour plot of the non-synchronized culture (Fig. 5b, left panel) and less than $45 \mathrm{~K}$ of FSC-A on the contour plot of the synchronized culture (Fig. 5b, right panel) resulted from different developmental stage of Plasmodium between the two separate cultures. To confirm heterogeneity in the non-synchronized culture, the $\mathrm{CV}$, which is a measure of relative variability, of FSC-A and SSC-A was statistically analysed. Despite statistical non-significance $(p>0.05)$, the non-synchronized culture tended to have a higher CV for both FSC-A and SSC-A (Fig. 5c), which confirms the relatively high heterogeneity of VSG+ cells. Thus, VSG-based flow cytometry is an effective alternative method for assessing synchronicity of $P$. falciparum development in erythrocytes.

To demonstrate the use of VSG for assessment of growth inhibitory effect of anti-malarial drug, malariainfected erythrocytes were incubated with $700 \mathrm{nM}$ DHA for $24 \mathrm{~h}$ following a standard assay [21]. The DHA- and DMSO-treated cells were stained with VSG and analysed by flow cytometry. In both the presence and absence of the drug, there were VSG+ cells exhibiting VSG ${ }^{\text {intermediate }}$ and $\mathrm{VSG}^{\text {low }}$ (Fig. 5d), which were likely to resemble trophozoite and ring form, respectively. These results showed that the number of VSG+ cells decreased following DHA treatment (Fig. 5d, right panel) compared to that of the DMSO-treated control cells (Fig. $5 \mathrm{~d}$, left panel and $p=0.02$ ). The majority of DHA-treated VSG+ cells appear as VSG ${ }^{\text {low }}$, implying 


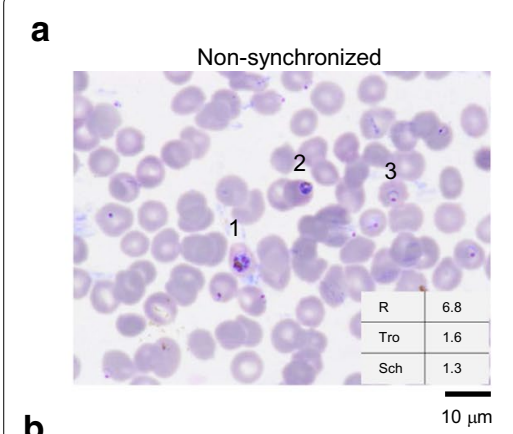

b Non-synchronized

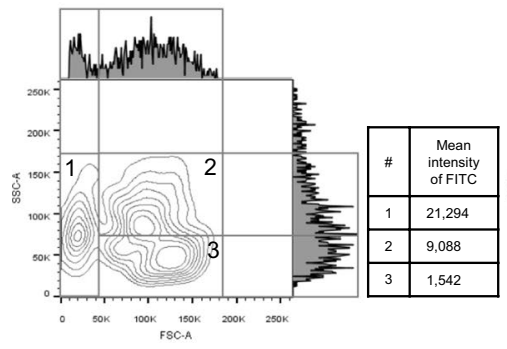

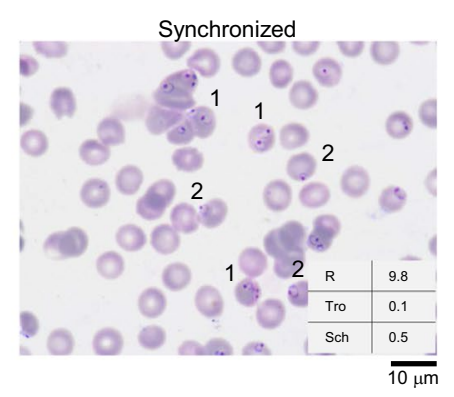

Synchronized

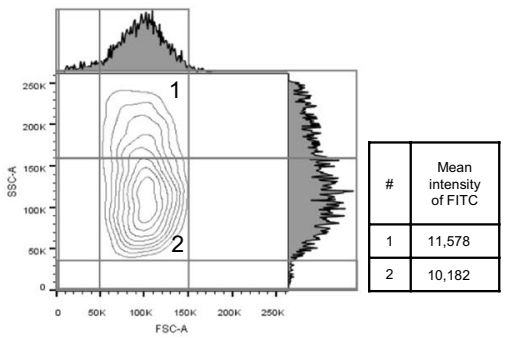

C
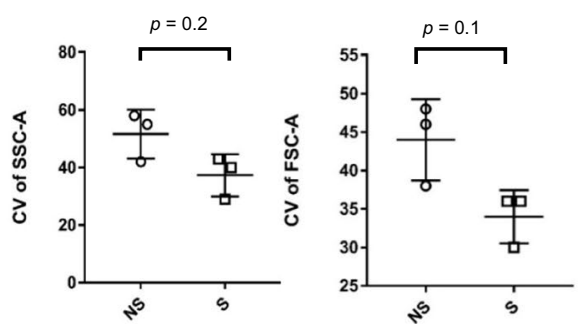

d
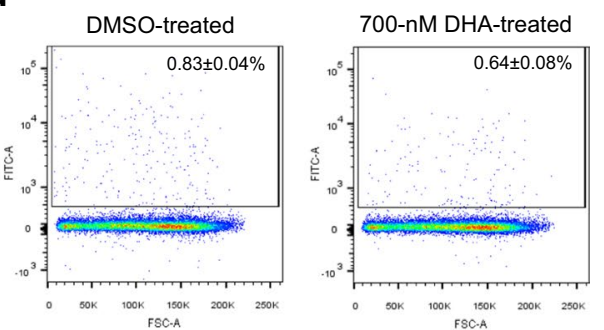

e

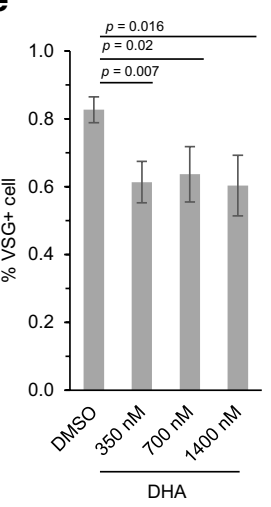

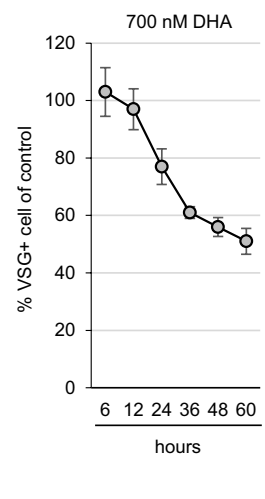

Fig. 5 Application of VSG-based flow cytometry in synchrony assessment and anti-malarial drug assay. a Microscopic images of $P$. falciparum-infected erythrocytes derived from non-synchronized and synchronized cultures and stained with Giemsa dye. b Flow cytometric profiles of VSG + cells are displayed according to FSC-A (X-axis) and SSC-A (Y-axis) in contour plots. Histograms of FSC-A and SSC-A are shown at the top and left side of the contour plots, respectively. There were at least three distinct populations observed in the non-synchronized culture (left panel), as follows: (1) cells having small size with various granularity; (2) cells having a relatively large size with high granularity; and, (3) cells having a relatively larger size with low granularity. There were two populations observed in the synchronized culture (right panel), as follows: (1) minor population with SSC-A higher than $160 \mathrm{~K}$, and (2) major population with SSC-A lower than $160 \mathrm{~K}$. c Graph of coefficient of variation of FSC-A and SSC-A. Dots represent three independent experiments. $\mathbf{d}$ P. falciparum-infected erythrocytes were treated with anti-malarial drug dihydroartemisinin (DHA) and subjected to VSG-based flow cytometric analysis. Synchronized ring forms were prepared. e Dose-dependent effect of DHA treatment on growth inhibition of P. falciparum. The infected erythrocytes were treated with 350, 700, and $1400 \mathrm{nM}$ of DHA for $24 \mathrm{~h}$ and subjected to VSG-based flow cytometric analysis. f Time-dependent effect of DHA treatment on growth inhibition of $P$. falciparum. After 6-h treatment with $700 \mathrm{nM}$ DHA, the infected erythrocytes were cultured in MCM without DHA and collected at 12, 24, 36, 48, and $60 \mathrm{~h}$ for VSG-based flow cytometric analysis. The number of VSG + cells in DMSO treatment was set as the control for each time point. The percentage of VSG+ cells in DHA treatment was compared to that observed in the control (Y-axis). X-axis indicates time of culture. Data represent mean \pm SD of three independent experiments. FSC-A, forward scatter-area; SSC-A, side scatter-area; NS, non-synchronized culture; S, synchronized culture; CV, coefficient of variation

that ring form was predominant. In contrast, both VSG $^{\text {intermediate }}$ and VSG ${ }^{\text {low }}$ cells were observed in the DMSO-treated control (Fig. 5d, left panel), implying that both ring from and trophozoite were present in the culture. Similar to 700-nM DHA treatment, number of VSG+ cells also decreased after treatment with 350 and $1400 \mathrm{nM}$ DHA for $24 \mathrm{~h}(p=0.007$ and 0.016 , respectively); however, there was no difference in number of VSG+ cells among doses (Fig. 5e). Moreover, VSG-based flow cytometry was able to access effect of 700-nM DHA in time-dependent manner (Fig. 5f). According to the VSG-based flow cytometric data, DHA likely inhibited parasite growth. Therefore, the VSG-based flow cytometric assay can be used as an alternative assay for assessment of $P$. falciparum growth in the presence of anti-malarial drug in vitro. 


\section{Discussion}

Many flow cytometric assays have been developed to detect the malaria parasite; however, these assays are complicated, time consuming, and/or insufficiently sensitive. In this study, VSG was used to detect and purify $P$. falciparum-infected erythrocytes using flow cytometry. VSG could enter and bind to the nucleic acid of ring form, trophozoite, schizont and early stage of gametocytes of $P$. falciparum growing in culture. Twenty-minute incubation at room temperature without fixation makes this method faster and simpler to perform than other malaria detection assays.

The degree of parasitaemia detected by VSG-based flow cytometry was different from the degree of parasitaemia detected by the standard microscopic method. This difference is likely due to the method of cell analysis. It is recommended that 10,000 cells be counted under the microscope in order to accurately quantify the number of malaria parasite-infected cells. In contrast, hundreds of thousands of cells could be analysed by flow cytometry. Thus, different percentages of infected cells could be expected from these two different methods. This difference between methods may be explained by the sensitivity of VSG dye and its ability to correctly identify different stages of $P$. falciparum. Further validation by comparing VSG to other florescent dyes is warranted. Even though the percentages of infected cells was different between methods, the VSGbased flow cytometric assay was found to be reliable for detecting the malaria parasite in a dose-dependent manner, and it was able to detect parasitized cells as low as $0.001 \%$, which is the detection threshold for the standard microscopic method [20].

In addition to the higher percentage of infected cells identified by the VSG method, the fixation-free flow cytometric method profiled in this report also provides morphologic information. This is the first study to report the high specificity of a method by showing the morphology of fluorochrome-binding cells. VSG+ cells were infected by $P$. falciparum, and all four major stages of malaria parasite could uptake VSG. Moreover, the intensity of VSG was found to be commensurate with the amount of DNA and RNA, and the VSG intensity of schizonts was higher than that of ring-shaped forms.

VSG is commercially available for nucleic acid detection in agarose gel electrophoresis and it is difficult for VSG to permeate cells; however, the reported data shows that VSG could enter P. falciparum-infected erythrocytes. It needs to be further investigated whether the permeability of VSG is due to increased membrane transport for nucleosides, amino acids, and carbohydrates, as described in previous studies [23, 24].
Compared to other DNA-binding fluorochromes, VSG is more suitable for analysing malaria parasites for the following reasons. First, VSG can rapidly enter cells and bind to nucleic acid at ambient temperature. As such, there is no need for cell permeabilization, which shortens the pre-flow cytometry process. Second, the fluorescence emission spectrum of VSG is similar to spectra of FITC, the most widely used fluorochrome. This factor facilitates application of VSG with other flow cytometry. Third and last, although Hoechst is frequently used as nuclear DNA stains, these fluorochrome probes cannot be used when the cytometer being used does not have a UV or 405-nm laser. Thus, VSG can be used as an alternative choice in the aforementioned setting.

VSG is an attractive alternative in flow cytometric assay due to its speed and ease of use compared to that of other DNA-binding fluorochrome probes, including hydroethidine [6], ethidium bromide [7], propidium iodide [8], SYBR Green I [9, 10], YOYO-1 [11], Hoechst 33258 [12], and Hoechst 33342 [13]. In comparison with Coriphosphine $\mathrm{O}$ [25], which is a fluorochrome activated by $488-\mathrm{nm}$ laser, the use of VSG is simpler and there is no requirement for incubation at $37{ }^{\circ} \mathrm{C}$ and cell washing prior to flow cytometric analysis. Moreover, the cost of VSG is lower than that of Coriphosphine O. Even though VSG is comparable to that of SYBR Green I relative to cost and ease of use (without cell fixation), the resolution of the different Plasmodium stages stained with SYBR Green I was not sharp [26], which had the effect of limiting parasite stage identification. Moreover, an additional step of cell fixation using paraformaldehyde (PFA) or 1\% glutaraldehyde was reported [9]. Importantly, VSG-based flow cytometric assay was able to detect a lower percentage of parasitaemia than Coriphosphine O and SYBR Green I. Despite these advantages, the protocol still requires further optimization, and a comparison with the aforementioned dyes is needed to draw definitive conclusions, regarding the advantages and disadvantages of each dye relative to VSG. Moreover, since VSG was originally used for DNA or RNA stain in agarose gel, and the dye can be activated using a 254-nm UV transilluminator, it may be possible that VSG-stained cells can be analysed using a UV laser-equipped flow cytometer and cell sorter.

The accurate detection and quantification of P. falciparum-infected erythrocytes using an automated Sysmex Haematology Analyzer XN-30 was recently reported [27, 28]. This automatic machine employs a 405-nm laser to detect cells that need to be partially lysed to increase the permeability of florescent dye before cell analysis. Thus, this method limits morphological observation of parasitized cells after cell analysis. Importantly, only a specific florescent dye can be used with this cell analyzer. In contrast, VSG is commercially available and is activated 
using a 488-nm excitation laser, which is one of the most common lasers built into cell analyzers, including: FACSCalibur, FACS Aria (BD Bioscineces), ZE5 Cell Analyzer (Bio-Rad), CellSimple ${ }^{\mathrm{TM}}$ Cell Analyzer (Cell Signaling Technology), and Guava ${ }^{\circledR}$ easyCyte ${ }^{\mathrm{TM}}$ Systems (Luminex). However, VSG-based flow cytometry is not able to distinguish early gametocyte stage in cultures containing schizonts.

Anti-malarial drug susceptibility assay is very useful for identifying pharmacologically active compounds, to monitor drug resistance, and to investigate the mechanisms underlying drug resistance. Effect of anti-malarial drugs is mainly characterized by the inhibition of parasite growth or maturation and multiplication. These parameters are often measured by uptake of radioisotope $\left[\mathrm{H}^{3}\right]$ hypoxanthine into nucleic acid [29], enzymatic assay of $P$. falciparum-specific lactate dehydrogenase [30], or detection of $P$. falciparum-specific antigen histidine-rich protein 2 in the culture [31]. The utility of VSG for assessing the pharmacologic effect of anti-malarial drugs on organism development was demonstrated.

\section{Conclusions}

Given a relative ease of use of fluorescent dyes, VSGbased flow cytometry may be an effective alternative assay for enumeration of parasitaemia, assessment of intraerythrocytic development and synchronicity, and anti-malarial drug effect.

\section{Supplementary information}

Supplementary information accompanies this paper at https://doi. org/10.1186/s12936-020-03156-1.

Additional file 1: Fig. S1. Effect of cell debris on analysis of VSG+ cells by flow cytometry. (A) In flow cytometry, cell debris has a lower level of forward scatter (FSC), and it can be observed at the bottom left corner of the density plot [32]. To remove cell debris, the threshold of FSC was set at 10,000 for all experiments. The presence of debris in three independent experiments was examined and shown. Cells having characteristics of FSC ${ }^{\text {low }}$ and that are located at bottom left (circles) of the density plot are considered debris. There is $0.64 \pm 0.2 \%$ of debris, suggesting that the majority of analysed cells are not debris. Moreover, the presence of cell debris in VSG + cells was checked by examining VSG + cells according to FSC-A and SSC-A (lower panels). There was $0.69 \pm 0.13 \%$ of VSG + cells that had characteristics of FSClow at bottom left corner of the density plot. Therefore, more than $95 \%$ of cells analysed by VSG-based flow cytometry were unlikely to be cell debris. (B) Morphology of cells having characteristics of FSClow $(<50$ K). P. falciparum-infected erythrocytes were subjected to flow cytometric analysis. The gated single cells were sorted according to intensity of FSC. There were ring forms and schizonts observed in the FSClow cells. Thus, inclusion of the FSC ${ }^{\text {low }}$ cells is required. Microscopic images were captured using a microscope with objective lens of 100x magnification.

Additional file 2: Fig. S2. VSG-based flow cytometric analysis of gametocyte. Early schizonts and mature schizonts (left panel) and early stage gametocytes (right panel) were observed in VSG ${ }^{\text {high }}$ fraction. In the VSG ${ }^{\text {high }}$ fraction, parasitized erythrocytes have granular distribution of haemozoin, resembling stage IB. Moreover, some were elongated and D-shaped within erythrocytes, which are key characteristics of stage II gametocytes.
Early schizonts having 2 and 6 divided nucleus, and mature schizonts consisting of 14 merozoites were also observed in the VSG ${ }^{\text {high }}$ fraction, whereas ring forms and trophozoites were observed in the VSG low and VSG intermediate fractions. Scale bar: $10 \mu \mathrm{m}$.

Additional file 3: Fig. S3. Giemsa stain of VSG+ cells sorted from nonsynchronized and synchronized culture of P. falciparum. (A) Morphology of three distinct populations observed in the non-synchronized culture (Fig. 5b, left panel): (1) cells having small size with various granularity (approximately 0-45 K of FSC-A, and 30-170 K of SSC-A); (2) cells having a relatively large size with high granularity (approximately $45-185 \mathrm{~K}$ of FSC-A, and 75-170 K of SSC-A); and, (3) cells having a relatively larger size with low granularity (approximately 45-185 K of FSC-A, and 20-75 K of SSC-A). Based on the intensity of VSG and microscopic images, the population number 1, 2, and 3 in the upper panel of Fig. $5 b$ are schizonts, trophozoites, and ring forms, respectively. (B) Morphology of one minor (indicated as 1) and one major (indicated as 2) population observed in the synchronized culture (Fig. 5b, right panel): (1) cells having high granularity (more than $160 \mathrm{~K}$ of SSC-A); and (2) cells having with low granularity (lower than $160 \mathrm{~K}$ of SSC-A). Both had a similar size (50-150 K of FSC-A). Based on microscopic images, majority of the VSG + cells with more than $160-K$ SSC-A are infected erythrocytes containing multiple ring forms, and they had VSG intensity of 11,578; whereas, the majority of VSG + cells with lower than 160 K SSC-A are infected erythrocytes with single ring forms and they had VSG intensity of 10,182.

\section{Abbreviations}

CV: Coefficient of variation; DHA: Dihydroartemisinin; DIC: Differential interference contrast; DMSO: Dimethyl sulfoxide; FBS: Fetal bovine serum; FSC-A: Forward scatter area; FSC-H: Forward scatter height; FSC-W: Forward scatter width; MCM: Malaria culture medium; NS: Non-synchronized; RPM: Revolutions per minute; RPMI: Roswell Park Memorial Institute; S: Synchronized; SD: Standard deviation; SSC-H: Side scatter height; SSC-W: Side scatter width; VSG: ViSafe Green.

\section{Acknowledgements}

The authors gratefully acknowledge Manachaya Pongwittayawakin and Thitisuda Sangkham for their valuable contributions to this study, and the Department of Anatomy, Faculty of Medicine Siriraj Hospital, Mahidol University for microscope technical support.

\section{Authors' contributions}

KK, AT, and DP conceived and designed the study. KK, NK, and DP performed the experiments, analysed and interpreted the data, and wrote the manuscript. All authors read and approved the final manuscript.

\section{Funding}

This research project was supported by a grant from the Siriraj Research Fund, Faculty of Medicine Siriraj Hospital, Mahidol University, Bangkok, Thailand (Grant No. [IO] R016233004), and by a Grant from Chulalongkorn University, Bangkok, Thailand.

\section{Availability of data and materials}

The datasets used and/or analysed during the current study are available from the corresponding authors on reasonable request.

\section{Ethics approval and consent to participate}

Ethical approval was obtained from the Ethical Review Committee for Research Involving Human Subjects in Research, Chulalongkorn University, in accordance with the International Conference on Harmonization-Good Clinical Practice (COA no. 128/2012). Written informed consent was obtained from each study participant before blood was obtained for malaria culture. The protocol for malaria culture was approved by the Siriraj Safety Risk Management Taskforce, Faculty of Medicine Siriraj Hospital, Mahidol University (COA no. SI 2018-011).

\section{Competing interests}

All authors declare no competing interests, no personal or professional conflicts of interest, and no financial support from the companies that produce and/or distribute the drugs, devices, or materials described in this report. 
Received: 20 November 2019 Accepted: 9 February 2020

Published online: 18 February 2020

\section{References}

1. Ashley EA, Pyae Phyo A, Woodrow CJ. Malaria. Lancet. 2018;391:1608-21.

2. van der Pluijm RW, Imwong M, Chau NH, Hoa NT, Thuy-Nhien NT, Thanh $\mathrm{NV}$, et al. Determinants of dihydroartemisinin-piperaquine treatment failure in Plasmodium falciparum malaria in Cambodia, Thailand, and Vietnam: a prospective clinical, pharmacological, and genetic study. Lancet Infect Dis. 2019;19:952-61.

3. Rieckmann KH, Campbell GH, Sax LJ, Mrema JE. Drug sensitivity of Plasmodium falciparum. An in-vitro microtechnique. Lancet. 1978;1:22-3.

4. Richards WH, Maples BK. Studies on Plasmodium falciparum in continuous cultivation. I. The effect of chloroquine and pyrimethamine on parasite growth and viability. Ann Trop Med Parasitol. 1979;73:99-108.

5. Nguyen-Dinh P, Trager W. Plasmodium falciparum in vitro: determination of chloroquine sensitivity of three new strains by a modified 48 -h test. Am J Trop Med Hyg. 1980:29:339-42.

6. Wyatt CR, GoffW, Davis WC. A flow cytometric method for assessing viability of intraerythrocytic hemoparasites. J Immunol Methods. 1991;140:23-30

7. Persson KE, Lee CT, Marsh K, Beeson JG. Development and optimization of high-throughput methods to measure Plasmodium falciparum-specific growth inhibitory antibodies. J Clin Microbiol. 2006;44:1665-73.

8. Pattanapanyasat $\mathrm{K}$, Thaithong S, Kyle DE, Udomsangpetch R, Yongvanitchit K, Hider RC, et al. Flow cytometric assessment of hydroxypyridinone iron chelators on in vitro growth of drug-resistant malaria. Cytometry. 1997;27:84-91.

9. Izumiyama S, Omura M, Takasaki T, Ohmae H, Asahi H. Plasmodium falciparum: development and validation of a measure of intraerythrocytic growth using SYBR Green I in a flow cytometer. Exp Parasitol. 2009;121:144-50.

10. Jang JW, Kim JY, Yoon J, Yoon SY, Cho CH, Han ET, et al. Flow cytometric enumeration of parasitemia in cultures of Plasmodium falciparum stained with SYBR Green I and CD235A. Sci World J. 2014;2014:536723.

11. Schuck DC, Ribeiro RY, Nery AA, Ulrich H, Garcia CR. Flow cytometry as a tool for analyzing changes in Plasmodium falciparum cell cycle following treatment with indol compounds. Cytometry A. 2011;79:959-64.

12. van Vianen $\mathrm{PH}$, van Engen $\mathrm{A}$, Thaithong $\mathrm{S}$, van der Keur $\mathrm{M}$, Tanke $\mathrm{HJ}$, van der Kaay $\mathrm{HJ}$, et al. Flow cytometric screening of blood samples for malaria parasites. Cytometry. 1993;14:276-80.

13. Dekel E, Rivkin A, Heidenreich M, Nadav Y, Ofir-Birin Y, Porat Z, et al. Identification and classification of the malaria parasite blood developmental stages, using imaging flow cytometry. Methods. 2017:112:157-66.

14. ViSafe Green Gel Stain. Vivantis. https://www.vivantechnologies.com/ index.php?option $=$ com_content\&view $=$ article\&id $=1193$ :visafe-green -gel-stain\&catid=83:nucleic-acid-dyes\&ltemid=128. Accessed 14 Nov 2019.

15. Trager W, Jensen JB. Human malaria parasites in continuous culture. Science. 1976;193:673-5.

16. Silamut K, White NJ. Relation of the stage of parasite development in the peripheral blood to prognosis in severe falciparum malaria. Trans R Soc Trop Med Hyg. 1993;87:436-43.
17. Lambros $C$, Vanderberg JP. Synchronization of Plasmodium falciparum erythrocytic stages in culture. J Parasitol. 1979;65:418-20.

18. Delves MJ, Straschil U, Ruecker A, Miguel-Blanco C, Marques S, Dufour $A C$, et al. Routine in vitro culture of $P$. falciparum gametocytes to evaluate novel transmission-blocking interventions. Nat Protoc. 2016;11:1668-80.

19. Talman AM, Domarle O, McKenzie FE, Ariey F, Robert V. Gametocytogenesis: the puberty of Plasmodium falciparum. Malar J. 2004;3:24

20. Mathison BA, Pritt BS. Update on malaria diagnostics and test utilization. J Clin Microbiol. 2017:55:2009-17.

21. Witkowski B, Amaratunga C, Khim N, Sreng S, Chim P, Kim S, et al. Novel phenotypic assays for the detection of artemisinin-resistant Plasmodium falciparum malaria in Cambodia: in-vitro and ex-vivo drug-response studies. Lancet Infect Dis. 2013;13:1043-9.

22. Herzenberg LA, Tung J, Moore WA, Herzenberg LA, Parks DR. Interpreting flow cytometry data: a guide for the perplexed. Nat Immunol. 2006;7:681-5.

23. Gero AM, Bugledich EM, Paterson AR, Jamieson GP. Stage-specific alteration of nucleoside membrane permeability and nitrobenzylthioinosine insensitivity in Plasmodium falciparum infected erythrocytes. Mol Biochem Parasitol. 1988:27:159-70.

24. Kirk K, Lehane AM. Membrane transport in the malaria parasite and its host erythrocyte. Biochem J. 2014;457:1-18.

25. Tiendrebeogo RW, Adu B, Singh SK, Dodoo D, Dziegiel MH, Mordmuller $B$, et al. High-throughput tri-colour flow cytometry technique to assess Plasmodium falciparum parasitaemia in bioassays. Malar J. 2014;13:412.

26. Russell B, Suwanarusk R, Borlon C, Costa FT, Chu CS, Rijken MJ, et al. A reliable ex vivo invasion assay of human reticulocytes by Plasmodium vivax. Blood. 2011;118:e74-81.

27. Tougan T, Toya Y, Uchihashi K, Horii T. Application of the automated haematology analyzer $\mathrm{XN}-30$ for discovery and development of anti-malarial drugs. Malar J. 2019;18:8.

28. Tougan T, Suzuki Y, Itagaki S, Izuka M, Toya Y, Uchihashi K, et al. An automated haematology analyzer $\mathrm{XN}$-30 distinguishes developmental stages of falciparum malaria parasite cultured in vitro. Malar J. 2018;17:59.

29. Chulay JD, Haynes JD, Diggs CL. Plasmodium falciparum: assessment of in vitro growth by [3H]hypoxanthine incorporation. Exp Parasitol. 1983;55:138-46.

30. Makler MT, Ries JM, Williams JA, Bancroft JE, Piper RC, Gibbins BL, et al. Parasite lactate dehydrogenase as an assay for Plasmodium falciparum drug sensitivity. Am J Trop Med Hyg. 1993:48:739-41.

31. Noedl $H$, Wernsdorfer WH, Miller RS, Wongsrichanalai C. Histidine-rich protein II: a novel approach to malaria drug sensitivity testing. Antimicrob Agents Chemother. 2002;46:1658-64.

32. MCFarland DC, Zhang C, Thomas HC, Ratliff TI. Confounding effects of platelets on flow cytometric analysis and cell-sorting experiments using blood-derived cells. Cytometry A. 2006;69:86-94.

\section{Publisher's Note}

Springer Nature remains neutral with regard to jurisdictional claims in published maps and institutional affiliations.

Ready to submit your research? Choose BMC and benefit from:

- fast, convenient online submission

- thorough peer review by experienced researchers in your field

- rapid publication on acceptance

- support for research data, including large and complex data types

- gold Open Access which fosters wider collaboration and increased citations

- maximum visibility for your research: over 100M website views per year

At $\mathrm{BMC}$, research is always in progress.

Learn more biomedcentral.com/submissions 